\title{
ON THE PROBLEM OF GROMOVA AND VASIL'EV ON INTEGRAL MEANS, AND YAMASHITA'S CONJECTURE FOR SPIRALLIKE FUNCTIONS
}

\author{
Saminathan Ponnusamy ${ }^{\dagger}$ and Karl-Joachim Wirths \\ Indian Statistical Institute (ISI), Chennai Centre, SETS, MGR Knowledge City \\ CIT Campus, Taramani, Chennai 600 113, India.; samy@isichennai.res.in, samy@iitm.ac.in \\ Technische Universität Braunschweig, Institut für Analysis und Algebra \\ 38106 Braunschweig, Germany; kjwirths@tu-bs.de
}

\begin{abstract}
In this article we consider some integral means problem for certain classes of univalent analytic functions, in particular for the class of the starlike functions of order $\beta$ and for the class of $\alpha$-spirallike functions of order $\beta$. Our investigation settles one of the open problems of Gromova and Vasil'ev. In addition, we solve another problem concerning area maximum property of $\alpha$-spirallike functions of order $\beta$ in the setting of Yamashita and hence, we find the solution to Yamashita's conjecture for certain Dirichlet-finite functions in a general form.
\end{abstract}

\section{Preliminaries and the main results}

In this paper, we investigate some families of univalent functions for which the range of each of its member function has a specific geometric property. We will be particularly interested in determining estimates for certain integral means for functions with geometric property. In this connection one of the classical results of Rogosinski [13] for subordination is useful. Using this, we prove a general result and a particular case solves one of the open problems of Gromova and Vasil'ev [5] on the best estimate for a special integral means for starlike functions of order $\beta$. Also, we prove Yamashita's conjecture on area maximum property for $\alpha$-spirallike functions of order $\beta$.

Let $\mathbf{D}:=\{z \in \mathbf{C}:|z|<1\}$ denote the open unit disk and $\mathcal{H}$ denote the class of all functions $f$ analytic in $\mathbf{D}$ with the topology of uniform convergence of compact subsets of $\mathbf{D}$ and $\mathcal{A}=\left\{f \in \mathcal{H}: f(0)=0\right.$ and $\left.f^{\prime}(0)=1\right\}$. For $f, g \in \mathcal{H}$, the symbol $f \prec g$ means that $f(z)=g(w(z))$, where $w(z)$ is analytic in $\mathbf{D}$ and $w(0)=0$ and $|w(z)|<1$ on $\mathbf{D}$. When $g$ is univalent in $\mathbf{D}, f \prec g$ if and only if $f(\mathbf{D}) \subset g(\mathbf{D})$ and $f(0)=g(0)$.

Lemma A. If $f \prec g$ and $g(z) \neq 0$ in $\mathbf{D}$, then, for each real $p$ and $0 \leq r<1$, we have

$$
\frac{1}{2 \pi} \int_{-\pi}^{\pi} \frac{d \theta}{\left|f\left(r e^{i \theta}\right)\right|^{2 p}} \leq \frac{1}{2 \pi} \int_{-\pi}^{\pi} \frac{d \theta}{\left|g\left(r e^{i \theta}\right)\right|^{2 p}}
$$

doi:10.5186/aasfm.2014.3922

2010 Mathematics Subject Classification: Primary 30C45, 30C70; Secondary 30H10, 33C05.

Key words: Analytic, univalent, convex, close-to-convex, starlike functions and spirallike functions, Dirichlet-finite, area integral, and Gaussian hypergeometric functions, Bessel's functions.

${ }^{\dagger}$ This author is on leave from the Department of Mathematics, Indian Institute of Technology Madras, Chennai-600 036, India. 
Proof. The case $p<0$ of (1) is a simple consequence of Rogosinski [13] subordination result. Since $g(z)$ is nonvanishing in the unit disk, we have

$$
\frac{1}{f(z)} \prec \frac{1}{g(z)}, \quad z \in \mathbf{D},
$$

and therefore, the inequality (1) for $p>0$ follows if we apply Rogosinski [13] subordination result to the last relation.

We shall use this lemma mainly for $p=1$, but we have stated it in this form as this will help to extend many results of Gromova and Vasil'ev [5]. However, we would like to point out that Lemma A gives generalizations of the some cases in Gromova and Vasil'ev [5], e.g. $\lambda_{2}=0$ in [5, Theorems 1,3,4]. We refer to Corollary 3. However, we are interested mainly in finding upper bounds in terms of the Taylor coefficients and this motivates the rest of the paper.

In this article, we consider the following two families of functions from $\mathcal{A}$. The first one is the class

$$
\mathcal{S}^{\star}(A, B)=\left\{f \in \mathcal{A}: \frac{z f^{\prime}(z)}{f(z)} \prec \frac{1+A z}{1+B z}, z \in \mathbf{D}\right\},
$$

where $-1 \leq B<A \leq 1$. For $\beta \in[0,1), \mathcal{S}^{\star}(1-2 \beta,-1)=$ : $\mathcal{S}^{\star}(\beta)$ denotes the usual normalized class of all (univalent) starlike functions of order $\beta$. The class $\mathcal{S}^{\star}(0):=\mathcal{S}^{\star}$ is the usual class of starlike functions, i.e. $f \in \mathcal{A}$ such that $f(\mathbf{D})$ is starlike with respect to the origin. Functions in $\mathcal{S}^{\star}(A, B)$ are included in the class $\mathcal{S}$ of univalent functions from $\mathcal{A}$ and the class $\mathcal{S}$ has been the central object in the study of geometric function theory.

The second one is the class $\mathcal{S}_{\alpha}(\beta)$ of functions, called $\alpha$-spirallike of order $\beta$. This is defined by

$$
\mathcal{S}_{\alpha}(\beta)=\left\{f \in \mathcal{A}: e^{-i \alpha} \frac{z f^{\prime}(z)}{f(z)} \prec \frac{e^{-i \alpha}+\left(e^{i \alpha}-2 \beta \cos \alpha\right) z}{1-z}, z \in \mathbf{D}\right\},
$$

where $\beta \in[0,1)$ and $\alpha \in(-\pi / 2, \pi / 2)$ (see [7]). Clearly, $\mathcal{S}_{\alpha}(\beta) \subset \mathcal{S}_{\alpha}(0)$. Functions in $\mathcal{S}_{\alpha}(0)$ are called $\alpha$-spirallike. The class $\mathcal{S}_{\alpha}(0)$ was introduced by Spaček [17] and the set $\mathcal{S} p=\cup\left\{\mathcal{S}_{\alpha}(0): \alpha \in(-\pi / 2, \pi / 2)\right\}$ is referred to us the class of spirallike functions and functions in $\mathcal{S} p$ are known to be univalent in D. As remarked in [7], spirallike functions have been used to obtain important counter-examples in geometric function theory (see also [4, p. 72 and Theorem 8.11]).

Also, it is clear that $\mathcal{S}^{\star}(\beta):=\mathcal{S}_{0}(\beta)$. We remark that the class of $\alpha$-spirallike functions is neither included in the Kaplan class $\mathcal{K}$ of close-to-convex functions nor includes it (see also [7]). A complete characterization of spirallike functions by means of subordination is given by Ruscheweyh [14].

In 2002, Gromova and Vasil'ev [5] considered the functional, called integral means,

$$
M\left(r, f, \lambda_{1}, \lambda_{2}\right)=\frac{1}{2 \pi} \int_{-\pi}^{\pi}\left|f\left(r e^{i \theta}\right)\right|^{\lambda_{1}}\left|f^{\prime}\left(r e^{i \theta}\right)\right|^{\lambda_{2}} d \theta \quad\left(z=r e^{i \theta} \in \mathbf{D}\right)
$$

where $\lambda_{1}, \lambda_{2} \in \mathbf{R}$. Choosing $\lambda_{1}=-2$ and $\lambda_{2}=0$ reduces to the integral means

$$
I_{1}(r, f):=M(r, f,-2,0)=\frac{1}{2 \pi} \int_{-\pi}^{\pi} \frac{d \theta}{\left|f\left(r e^{i \theta}\right)\right|^{2}}
$$


The estimates of $I_{1}$ and the mean $I_{2}$, where $I_{2}(r, f):=M(r, f, 0,-2)$, have received special attention. One of the reasons for these choices is backgrounded by some problems in planar fluid mechanics, where these functionals are participating in isoperimetric problems for moving phase domains, e.g. [19] and [20]. In [5], the authors obtained that if $f \in \mathcal{S}^{\star}(\beta)$, then the estimate

$$
L_{1}(r, f):=r^{2} I_{1}(r, f) \leq 1+4(1-\beta)^{3} r^{2}+(1-\beta) r^{2}, \quad r \in(0,1],
$$

holds and the inequality is sharp only for $\beta=0$ and $\beta=1 / 2$. It was also remarked that a sharp estimate is still unknown (see [5, p. 565]). Furthermore, not much is known concerning the estimate for $L_{1}(r, f)$ for many geometric classes of functions from $\mathcal{S}$. One of our aims is to state the following sharp estimate as a corollary to a main result and hence, the open problem of Gromova and Vasil'ev [5] is settled.

Corollary 1. Let $f \in \mathcal{S}^{\star}(\beta)$ for $0 \leq \beta<1$. Then

$$
L_{1}(r, f):=r^{2} I_{1}(r, f) \leq \frac{\Gamma(5-4 \beta)}{\Gamma^{2}(3-2 \beta)}, \quad r \in(0,1] .
$$

In particular, $L_{1}(r, f) \leq 6$ for $f \in \mathcal{S}^{\star}$. Both inequalities are sharp.

From Corollary 1 , it follows that $L_{1}(r, f) \leq 2$ for $f \in \mathcal{C}$. Here $\mathcal{C}$ denotes the class of convex functions from $\mathcal{S}$. This is because $\mathcal{C} \subsetneq \mathcal{S}^{\star}(1 / 2)$ and

$$
L_{1}(r, f) \leq 2 \text { for } f \in \mathcal{S}^{\star}(1 / 2) .
$$

As remarked in [11], many useful probabilities can be expressed in terms of the Taylor coefficients of $z / f(z)$, its derivatives, or their combinations and as a consequence of it, certain known inequalities for such combinations allow us to find explicit estimates for probabilities. We refer to $[6,8,9,15,16]$ where one can find many interesting applications in the theory of analytic fixed point functions and even in questions in probability.

To state our first main result, we need some preparation. Let ${ }_{2} F_{1}(a, b ; c ; z):=$ $F(a, b ; c ; z)$ denote the Gaussian hypergeometric function defined by

$$
F(a, b ; c ; z):=1+\sum_{k=1}^{\infty} \frac{(a)_{k}(b)_{k}}{(c)_{k}} \frac{z^{k}}{k !}, \quad z \in \mathbf{D},
$$

where $a, b, c$ are complex numbers such that $c \neq-m, m=0,1,2,3, \ldots,(a)_{0}=1$ for $a \neq 0$ and $(a)_{k}$ denotes the Pochhammer symbol $(a)_{k}:=a(a+1) \cdots(a+k-1)$ for $k \in \mathbf{N}$. In general, $a, b$ and $c$ are complex numbers with $c \neq 0,-1,-2, \ldots$ Note that if either $a=0$ or $b=0$, then $F(a, b ; c ; z)=1$. Basic information about Gaussian hypergeometric functions may be obtained from standard text books, for example the books of Temme [18] and Anderson et al. [1]. We see that $(a)_{n}=\Gamma(a+n) / \Gamma(a)$ and, in the exceptional case $c=-m, m=0,1,2,3, \ldots$, the function $F(a, b ; c ; z)$ is clearly defined even if $a=-j$ or $b=-j$, where $j=0,1,2, \ldots$ and $j \leq m$. The following well-known Gauss formula is also used to simplify certain expressions:

$$
F(a, b ; c ; 1)=\frac{\Gamma(c) \Gamma(c-a-b)}{\Gamma(c-a) \Gamma(c-b)}<\infty \quad \text { for } \operatorname{Re} c>\operatorname{Re}(a+b) .
$$

We now state our first main result from which one can obtain results for several special cases. 
Theorem 1. For $-1 \leq B<A \leq 1$, let $f \in \mathcal{S}^{\star}(A, B)$. Then we have

$$
L_{1}(r, f):=r^{2} I_{1}(r, f) \leq \begin{cases}F\left((A / B)-1,(A / B)-1 ; 1 ;|B|^{2}\right) & \text { for } B \neq 0, \\ J_{0}(2 i|A|) & \text { for } B=0,\end{cases}
$$

where $J_{0}(z)$ is the Bessel function of order zero. Both inequalities are sharp.

For the definition of $J_{0}(z)$, we refer to [18, Eqn. (9.14), p. 228].

Proof of Corollary 1. Proof follows by choosing $A=1-2 \beta$ and $B=-1$ in Theorem 1. Thus, we have

$$
L_{1}(r, f):=r^{2} I_{1}(r, f) \leq F\left((A / B)-1,(A / B)-1 ; 1 ;|B|^{2}\right)=F(2 \beta-2,2 \beta-2 ; 1 ; 1) .
$$

The desired conclusion follows if we use the well-known Gauss formula (2).

In addition to this case, the following special case may be worth mentioning.

Corollary 2. If $f \in \mathcal{S}^{\star}(A,-A)$ for $0<A \leq 1$, then we have the sharp inequality $L_{1}(r, f) \leq 1+4 A^{2}+A^{4}$. In particular, $L_{1}(r, f) \leq 6$ for $f \in \mathcal{S}^{\star}(1,-1):=\mathcal{S}^{\star}$.

As remarked in the beginning, we may now include an application of Lemma A.

Corollary 3. Let $f \in \mathcal{S}^{\star}(A, B)$ for some $-1 \leq B<A \leq 1$. Then for $\lambda \in \mathbf{R}$, we have

$$
\frac{1}{2 \pi} \int_{-\pi}^{\pi}\left|f\left(r e^{i \theta}\right)\right|^{\lambda} d \theta \leq \frac{r^{\lambda}}{2 \pi} \int_{-\pi}^{\pi} \frac{d \theta}{\left|q_{A, B}\left(r e^{i \theta}\right)\right|^{\lambda}}, \quad 0<r<1,
$$

where $q_{A, B}(z)$ is defined by $(6)$. The estimate is sharp.

In particular, for $f \in \mathcal{S}^{\star}(\beta)(0 \leq \beta<1)$, one has

$$
\frac{1}{2 \pi} \int_{-\pi}^{\pi}\left|f\left(r e^{i \theta}\right)\right|^{\lambda} d \theta \leq \frac{r^{\lambda}}{2 \pi} \int_{-\pi}^{\pi} \frac{d \theta}{\left|1-r e^{i \theta}\right|^{2(1-\beta) \lambda}}, \quad 0<r<1 .
$$

Moreover, for $2(1-\beta) \lambda>1$, we have

$$
\frac{1}{2 \pi} \int_{-\pi}^{\pi}\left|f\left(r e^{i \theta}\right)\right|^{\lambda} d \theta=O\left((1-r)^{1-2(1-\beta) \lambda-\epsilon}\right) \quad \text { as } r \rightarrow 1^{-}
$$

for every $\epsilon>0$.

In the following theorem we prove the analog of Theorem 1 for functions that are $\alpha$-spirallike of order $\beta$.

Theorem 2. Let $f \in \mathcal{S}_{\alpha}(\beta)$ for some $0 \leq \beta<1$ and $\alpha \in(-\pi / 2, \pi / 2)$. Then we have

$$
L_{1}(r, f):=r^{2} I_{1}(r, f) \leq 1+\sum_{k=1}^{\infty}\left|\left(\begin{array}{l}
\gamma \\
k
\end{array}\right)\right|^{2}
$$

where $\gamma=2(1-\beta) e^{i \alpha} \cos \alpha$. In particular, $L_{1}(r, f) \leq 6$ for $f \in \mathcal{S}^{\star}(1,-1):=\mathcal{S}^{\star}$. Both inequalities are sharp.

Proofs of Theorems 1 and 2 will be presented in Section 2 .

For $g \in \mathcal{H}$, we denote the image of $|z|<r$ under $g$ by $\Delta(r, g)$, where $0<r \leq 1$. Thus for $g(z)=\sum_{n=0}^{\infty} b_{n} z^{n}$, we have

$$
\Delta(r, g)=\iint_{|z|<r}\left|g^{\prime}(z)\right|^{2} d x d y=\pi \sum_{n=1}^{\infty} n\left|b_{n}\right|^{2} r^{2 n} \quad(z=x+i y) .
$$


We call $g$ a Dirichlet-finite function whenever $\Delta(1, g)$, the area covered by the mapping $z \rightarrow g(z)$ for $|z|<1$, is finite. Yamashita [21, p. 439] conjectured that

$$
\max _{f \in \mathcal{C}} \Delta\left(r, \frac{z}{f(z)}\right)=\pi r^{2}, \text { for } 0<r \leq 1,
$$

where the maximum is attained only by the rotations of the function $j(z)=z /(1-z)$. Recently, this conjecture was settled by Obradović et al. in [11, Theorem 3] in the following general form.

Theorem B. Let $f \in \mathcal{S}^{\star}(\beta)$ for some $0 \leq \beta<1$. Then we have

$$
\max _{f \in \mathcal{S}^{\star}(\beta)} \Delta\left(r, \frac{z}{f(z)}\right)=A_{\beta}(r), \text { for } 0<r \leq 1,
$$

where $A_{\beta}(r)=4 \pi(1-\beta)^{2} r^{2} F\left(2 \beta-1,2 \beta-1 ; 2 ; r^{2}\right)$, and the maximum is attained by the rotations of $f_{\beta}(z)=z /(1-z)^{2(1-\beta)}$.

Next we state the following general result which proves Yamashita's extremal problem for the class $\mathcal{S}_{\alpha}(\beta)$.

Theorem 3. Let $f \in \mathcal{S}_{\alpha}(\beta)$, i.e., $f$ is $\alpha$-spirallike function of order $\beta(0 \leq \beta<1$ and $\alpha \in(-\pi / 2, \pi / 2))$. Then we have

$$
\max _{f \in \mathcal{S}_{\alpha}(\beta)} \Delta\left(r, \frac{z}{f(z)}\right)=A_{\alpha, \beta}(r), \text { for } 0<r \leq 1,
$$

where $A_{\alpha, \beta}(r)=\pi|\gamma|^{2} r^{2} F\left(1-\gamma, 1-\bar{\gamma} ; 2 ; r^{2}\right)$ and the maximum is attained by the rotations of $f_{\alpha, \beta}(z)=z /(1-z)^{\gamma}$. Here $\gamma=2(1-\beta) e^{i \alpha} \cos \alpha$.

In [11], the authors have proved Theorem 3 for $\alpha=0$ and thus, Theorem 3 implies Theorem B if we take $\alpha=0$. Consequently, we just provide some basic steps of the proof of Theorem 3 in Section 3. Also, it is important to observe that functions in $\mathcal{S}_{\alpha}(\beta)$ are not necessarily belonging to $\mathcal{S}^{\star}(\beta)$. This observation shows that Theorem 3 covers many other situations. However, it is an open problem to derive Yamashita's conjecture for convex functions of order $\beta$ and more generally, for functions in the class $\mathcal{S}^{\star}(A, B)$ and also for the class of functions $f$ for which $z f^{\prime}(z)$ in $\mathcal{S}^{\star}(A, B)$.

We end this section with the following special case $\beta=0$ of Theorem 3 .

Corollary 4. Let $f \in \mathcal{S}_{\alpha}(0)$, i.e., $f$ is $\alpha$-spirallike function for some $\alpha \in$ $(-\pi / 2, \pi / 2)$. Then we have

$$
\max _{f \in \mathcal{S}_{\alpha}(0)} \Delta\left(r, \frac{z}{f(z)}\right)=A_{\alpha, 0}(r), \text { for } 0<r \leq 1,
$$

where

$$
A_{\alpha, 0}(r) \leq A_{\alpha, 0}(1)=\frac{\pi \Gamma\left(4 \cos ^{2} \alpha\right)}{\Gamma\left(2 e^{i \alpha} \cos \alpha\right) \Gamma\left(2 e^{-i \alpha} \cos \alpha\right)}
$$

and the maximum is attained by the rotations of $f_{\alpha, 0}(z)=z /(1-z)^{2 e^{i \alpha} \cos \alpha}$.

\section{Solution to the problem of Gromova and Vasil'ev}

Proof of Theorem 1. Let $f \in \mathcal{S}^{\star}(A, B)$ and $p(z)=z / f(z)$. Then $p(z)$ is analytic in $\mathbf{D}, p(0)=1, p(z) \neq 0$ in $\mathbf{D}$ and

$$
\frac{z f^{\prime}(z)}{f(z)}=1-\frac{z p^{\prime}(z)}{p(z)} \prec \frac{1+A z}{1+B z}, \quad z \in \mathbf{D},
$$


so that

$$
\frac{z p^{\prime}(z)}{p(z)} \prec \frac{-(A-B) z}{1+B z}=: \varphi(z), \quad z \in \mathbf{D} .
$$

Note that $\varphi(z)$ is convex in $\mathbf{D}$ and $\varphi(0)=0$. It follows that (see for example [10, Corollary 3.1d.1, p. 76])

$$
p(z) \prec q_{A, B}(z)=\exp \left(\int_{0}^{z} \frac{\varphi(t)}{t} d t\right) .
$$

It is a simple exercise to compute that

$$
q_{A, B}(z)= \begin{cases}(1+B z)^{1-(A / B)} & \text { for } B \neq 0, \\ e^{-A z}=\lim _{B \rightarrow 0}(1+B z)^{1-(A / B)} & \text { for } B=0,\end{cases}
$$

or equivalently

$$
q_{A, B}(z)= \begin{cases}F(1, \delta ; 1, B z) & \text { for } B \neq 0 \\ e^{-A z} & \text { for } B=0\end{cases}
$$

with $\delta=(A / B)-1$. In particular, for $0<A \leq 1$, we have $q_{A,-A}(z)=(1-A z)^{2}$.

We now apply to (5) the theorem of Rogosinski [13]. According to this if

$$
p(z)=\frac{z}{f(z)}=\sum_{k=0}^{\infty} b_{k} z^{k}
$$

and $q_{A, B}(z)=\sum_{k=0}^{\infty} c_{k} z^{k}$ are two analytic functions such that $p(z) \prec q_{A, B}(z)$, then

$$
\sum_{k=0}^{n}\left|b_{k}\right|^{2} r^{2 k} \leq \sum_{k=0}^{n}\left|c_{k}\right|^{2} r^{2 k}
$$

for each $n \in \mathbf{N}$ and for each $r \in(0,1)$. Thus, by (5) we have

$$
\sum_{k=0}^{n}\left|b_{k}\right|^{2} r^{2 k} \leq \begin{cases}\sum_{k=0}^{n}\left(\frac{(\delta)_{k}}{k !}\right)^{2}|B|^{2 k} r^{2 k} & \text { for } B \neq 0 \\ \sum_{k=0}^{n} \frac{|A|^{2 k}}{(k !)^{2}} r^{2 k}<J_{0}(2 i|A| r) & \text { for } B=0\end{cases}
$$

where $J_{0}$ is the Bessel function of order zero. In this inequality, we can take $r=1$ and allow $n \rightarrow \infty$ (as the above estimate is finite). This gives the inequality

$$
\sum_{k=0}^{\infty}\left|b_{k}\right|^{2} \leq \begin{cases}F\left(\delta, \delta ; 1 ;|B|^{2}\right) & \text { for } B \neq 0 \\ J_{0}(2 i|A|) & \text { for } B=0 .\end{cases}
$$

Finally, the desired conclusion follows from the last estimates and the following

$$
r^{2} I_{1}(r, f)=\frac{1}{2 \pi} \int_{-\pi}^{\pi} \frac{r^{2}}{\left|f\left(r e^{i \theta}\right)\right|^{2}} d \theta=1+\sum_{k=1}^{\infty}\left|b_{k}\right|^{2} r^{2 k} \leq 1+\sum_{k=1}^{\infty}\left|b_{k}\right|^{2} .
$$

Equality occurs in the above inequalities if $f(z)=z / q_{A, B}(z)$.

Proof of Corollary 3. Let $f \in \mathcal{S}^{\star}(A, B)$ for some $-1 \leq B<A \leq 1$. Since $\mathcal{S}^{\star}(A, B) \subset \mathcal{S}^{\star}$ and $f$ is univalent in $\mathbf{D}$, we obtain that $f(z) \neq 0$ in $0<|z|<1$. Also, 
by (6), we have

$$
\sqrt{\frac{z}{f(z)}} \prec \sqrt{\frac{z}{q(z)}}, \quad q(z)= \begin{cases}\frac{z}{(1+B z)^{1-(A / B)}} & \text { for } B \neq 0, \\ z e^{A z} & \text { for } B=0 .\end{cases}
$$

The desired conclusion follows from Lemma A and the fact that, for $p>1$,

$$
\int_{-\pi}^{\pi} \frac{d \theta}{\left|1-r e^{i \theta}\right|^{p}}=O\left((1-r)^{1-p}\right) \quad \text { as } r \rightarrow 1^{-} .
$$

The special case follows if we choose $A=1-2 \beta$ and $B=-1$.

Proof of Theorem 2. Let $f \in \mathcal{S}_{\alpha}(\beta)$. Then

$$
e^{-i \alpha} \frac{z f^{\prime}(z)}{f(z)} \prec \frac{e^{-i \alpha}+\left(e^{i \alpha}-2 \beta \cos \alpha\right) z}{1-z}, \quad z \in \mathbf{D},
$$

and it is a simple exercise to see that the function $F$ defined by

$$
F(z)=z\left(\frac{f(z)}{z}\right)^{2 /\left[(1-\beta)\left(1+e^{2 i \alpha}\right)\right]}
$$

belongs to $\mathcal{S}^{\star}$. Further, $F \in \mathcal{S}^{\star}$ implies that

$$
\frac{z}{F(z)}=\left(\frac{z}{f(z)}\right)^{2 /\left[(1-\beta)\left(1+e^{2 i \alpha}\right)\right]} \prec(1-z)^{2},
$$

or equivalently,

$$
\frac{z}{f(z)} \prec \frac{z}{f_{\alpha, \beta}(z)}:=(1-z)^{(1-\beta)\left(1+e^{2 i \alpha}\right)}=(1-z)^{\gamma},
$$

where $\gamma=2(1-\beta) e^{i \alpha} \cos \alpha$. It is easy to see that $f_{\alpha, \beta} \in \mathcal{S}_{\alpha}(\beta)$. As in the proof of Theorem 1, this gives the inequality

$$
1+\sum_{k=1}^{\infty}\left|b_{k}\right|^{2} \leq 1+\sum_{k=1}^{\infty}\left|\left(\begin{array}{l}
\gamma \\
k
\end{array}\right)\right|^{2}
$$

where $\frac{z}{f(z)}=1+\sum_{k=1}^{\infty} b_{k} z^{k}$. It follows that for $f \in \mathcal{S}_{\alpha}(\beta)$,

$$
L_{1}(r, f) \leq 1+\sum_{k=1}^{\infty}\left|b_{k}\right|^{2} \leq 1+\sum_{k=1}^{\infty}\left|\left(\begin{array}{l}
\gamma \\
k
\end{array}\right)\right|^{2}
$$

and the desired conclusion follows. Equality occurs in the above inequalities if $f(z)=$ $f_{\alpha, \beta}(z)$.

\section{Yamashita's conjecture for spirallike functions}

In order to prove the Yamashita's conjecture for the class $\mathcal{S}_{\alpha}(\beta)$, we need to investigate the function

$$
f_{\alpha, \beta}(z)=\frac{z}{(1-z)^{\gamma}}=\frac{z}{F(1,-\gamma ; 1 ; z)},
$$

where $\gamma=2(1-\beta) e^{i \alpha} \cos \alpha$. As in [11], it follows easily that

$$
\Delta\left(r, \frac{z}{f_{\alpha, \beta}(z)}\right)=\pi \sum_{n=1}^{\infty} n\left|\frac{(-\gamma)_{n}}{(1)_{n}}\right|^{2} r^{2 n}=\pi|\gamma|^{2} r^{2} F\left(1-\gamma, 1-\bar{\gamma} ; 2 ; r^{2}\right)=: A_{\alpha, \beta}(r) .
$$


Also, for $r \in(0,1]$, we observe that

$$
A_{\alpha, \beta}(r)=\pi|\gamma|^{2} \sum_{n=1}^{\infty} \frac{\left|(1-\gamma)_{n-1}\right|^{2}}{(2)_{n-1}(1)_{n-1}} r^{2 n}
$$

Since the coefficients of the series on the right are all positive (except for the case $\alpha=0$ and $\beta=1 / 2$ for which $\left.A_{0,1 / 2}(r)=\pi r^{2}\right)$, the function $A_{\alpha, \beta}(r)$ is an increasing and convex function of the real variable $r, 0<r \leq 1$. This observation shows that

$$
A_{\alpha, \beta}(r) \leq A_{\alpha, \beta}(1)=\pi|\gamma|^{2} F(1-\gamma, 1-\bar{\gamma} ; 2 ; 1) .
$$

According to the well-known Gauss formula (2), the last expression becomes

$$
A_{\alpha, \beta}(r) \leq A_{\alpha, \beta}(1)=\pi\left(\frac{\Gamma(2 \operatorname{Re} \gamma)}{\Gamma(\gamma) \Gamma(\bar{\gamma})}\right)
$$

Proof of Theorem 3. Since the theorem for the case of $\alpha=0$ has been proved in [11], we may exclude the case $\alpha=0$ throughout the discussion below. Let $f \in \mathcal{S}_{\alpha}(\beta)$ for some $0 \leq \beta<1$ and $\alpha \in(-\pi / 2, \pi / 2)$. Set further

$$
g(z)=\frac{z}{f(z)}=1+\sum_{k=1}^{\infty} b_{k} z^{k} \quad \text { and } \quad(1+z)^{2(1-\beta) e^{i \alpha} \cos \alpha}=1+\sum_{k=1}^{\infty}(-1)^{k} c_{k} z^{k}
$$

and $r \in(0,1)$. As in [11], it suffices to show that for each $N \in \mathbf{N}$,

$$
\sum_{k=1}^{N} k\left|b_{k}\right|^{2} r^{2 k} \leq \sum_{k=1}^{N} k\left|c_{k}\right|^{2} r^{2 k}
$$

holds. In order to prove the inequality (7), we begin to observe that

$$
e^{-i \alpha} \frac{z g^{\prime}(z)}{g(z)}=e^{-i \alpha}-e^{-i \alpha} \frac{z f^{\prime}(z)}{f(z)}, \quad z \in \mathbf{D} .
$$

Since $f \in \mathcal{S}_{\alpha}(\beta)$, there exists an analytic function $\omega: \mathbf{D} \rightarrow \overline{\mathbf{D}}$ such that

$$
e^{-i \alpha} \frac{z g^{\prime}(z)}{g(z)}=2(1-\beta) \cos \alpha \frac{z \omega(z)}{1+z \omega(z)}, \quad z \in \mathbf{D},
$$

or, equivalently

$$
g^{\prime}(z)=\left(\gamma g(z)-z g^{\prime}(z)\right) \omega(z), \quad z \in \mathbf{D}
$$

where $\gamma=2(1-\beta) e^{i \alpha} \cos \alpha$. In terms of power series, this is same as

$$
\sum_{k=1}^{\infty} k b_{k} z^{k-1}=\left(\gamma+\sum_{k=1}^{\infty}(\gamma-k) b_{k} z^{k}\right) \omega(z), \quad z \in \mathbf{D} .
$$

But then by the method of Clunie [2] (see also [3, 12, 13]), it follows that

$$
\sum_{k=1}^{n-1}\left|b_{k}\right|^{2} r^{2(k-1)}\left(k^{2}-|k-\gamma|^{2} r^{2}\right)+\left|b_{n}\right|^{2} r^{2(n-1)} n^{2} \leq|\gamma|^{2}
$$

which upon multiplication by $r^{2}$ shows that

$$
\sum_{k=1}^{n-1}\left|b_{k}\right|^{2} r^{2 k}\left(k^{2}-|k-\gamma|^{2} r^{2}\right)+\left|b_{n}\right|^{2} r^{2 n} n^{2} \leq r^{2}|\gamma|^{2} .
$$


As the function $b(z)=(1+z)^{\gamma}$ satisfies the differential equation

$$
b^{\prime}(z)=\gamma b(z)-z b^{\prime}(z), \quad z \in \mathbf{D},
$$

it is clear that in the inequalities (9) equality is attained for $b_{k}=(-1)^{k} c_{k}$. We only have to give the crucial steps and appropriate replacements from [11] as the rest of proof follows from there.

As in [11], we consider the inequalities (9) for $n=1, \ldots, N$ and multiply the $n$-th inequality by a factor $\lambda_{n, N}$ such that the addition of the modified inequalities results in the left hand side of (7). For the calculation of the factors $\lambda_{n, N}$, we get the following system of linear equations

$$
k=k^{2} \lambda_{k, N}+\sum_{n=k+1}^{N} \lambda_{n, N}\left(k^{2}-|k-\gamma|^{2} r^{2}\right), \quad k=1, \ldots, N,
$$

which may be rewritten as

$$
\frac{1}{k}=\lambda_{k, N}+(1-a(k)) \sum_{n=k+1}^{N} \lambda_{n, N}, \quad k=1, \ldots, N,
$$

with $a(k)=\left|1-\frac{\gamma}{k}\right|^{2} r^{2}$. Here $k=N$ gives $\lambda_{N, N}=1 / N$. Again, since the matrix of this system is an upper triangular matrix with positive integers as diagonal elements, the solution of this system is uniquely determined. Following the method of proof of [11, Lemma 2], this leads to the following formula for $k \leq N-1$ :

$$
\lambda_{k, N}=\lambda_{k, N-1}-\frac{1}{N}(1-a(k)) \prod_{m=k+1}^{N-1} a(m) .
$$

In order to present a self-contained proof of it without using Cramer's rule, we would like to include here a direct and an alternate proof of the last relation. In fact a comparison of (10) by itself with $N$ replaced by $N-1$ shows that

$$
\lambda_{k, N-1}-\lambda_{k, N}=(1-a(k))\left[\sum_{n=k+1}^{N-1}\left(\lambda_{n, N}-\lambda_{n, N-1}\right)+\frac{1}{N}\right]
$$

which for $k=N-1$ means

$$
\lambda_{N-1, N-1}-\lambda_{N-1, N}=\frac{1}{N}(1-a(N-1))=\frac{1}{N}\left(1-\left|1-\frac{\gamma}{N-1}\right|^{2} r^{2}\right) .
$$

Now, we assume that (11) is true for $k=l$ with $l=N-1, N-2, \ldots, k+1$. That is,

$$
\lambda_{l, N}-\lambda_{l, N-1}=-\frac{1}{N}(1-a(l)) T_{l+1}, \quad T_{l+1}=\prod_{m=l+1}^{N-1} a(m),
$$

for $l=N-1, N-2, \ldots, k+1$. Then, by (12), we find that

$$
\begin{aligned}
\lambda_{k, N-1}-\lambda_{k, N} & =(1-a(k))\left[\sum_{n=k+1}^{N-1}\left(\lambda_{n, N}-\lambda_{n, N-1}\right)+\frac{1}{N}\right] \\
& =(1-a(k))\left[-\frac{1}{N} \sum_{n=k+1}^{N-1}(1-a(n)) T_{n+1}+\frac{1}{N}\right]
\end{aligned}
$$




$$
=\frac{1}{N}(1-a(k))\left[-\left(1-T_{k+1}\right)+1\right]=\frac{1}{N}(1-a(k)) T_{k+1}
$$

and thus, we have proved (11).

We see that for fixed $k \in \mathbf{N}, N \geq k$, the sequence $\left\{\lambda_{k, N}\right\}$ is a strictly decreasing sequence with

$$
\lambda_{k}:=\lim _{N \rightarrow \infty} \lambda_{k, N}=\frac{1}{k}-\left(1-\left|1-\frac{\gamma}{k}\right|^{2} r^{2}\right) S_{k}
$$

where

$$
S_{k}=\sum_{n=k+1}^{\infty} \frac{1}{n} \prod_{m=k+1}^{n-1}\left(\left|1-\frac{\gamma}{m}\right|^{2} r^{2}\right), \quad \text { for } k \in \mathbf{N} \cup\{0\} .
$$

To prove that $\lambda_{k, N}>0$ for all $N \in \mathbf{N}, 1 \leq k \leq N$, it is sufficient to prove $\lambda_{k} \geq 0$ for $k \in \mathbf{N}$. The last step concerning non-negativity of the multipliers $\lambda_{k}$ for $k \in \mathbf{N}$ is equivalent to proving that

$$
S_{k} \leq \frac{1}{k\left(1-\left|1-\frac{\gamma}{k}\right|^{2} r^{2}\right)}
$$

But before that we want to remark that the proof of the said inequality is sufficient for the proof of the assertion of the theorem, since, as we remarked above, equality is attained for $b_{k}=(-1)^{k} c_{k}$.

Following the ideas from [11, Proof of Lemma 2], the identity

$$
S_{k-1}=\frac{1}{k}+\left|1-\frac{\gamma}{k}\right|^{2} r^{2} S_{k}
$$

implies that (14) is equivalent with

$$
S_{k-1} \leq \frac{1}{k\left(1-\left|1-\frac{\gamma}{k}\right|^{2} r^{2}\right)}
$$

To prove (15) we use the inequality

$$
\frac{1}{n\left(1-\left|1-\frac{\gamma}{n}\right|^{2} r^{2}\right)}>\frac{1}{(n+1)\left(1-\left|1-\frac{\gamma}{n+1}\right|^{2} r^{2}\right)}
$$

and the identity

$$
\frac{1}{n\left(1-\left|1-\frac{\gamma}{n}\right|^{2} r^{2}\right)}=\frac{1}{n}+\frac{\left|1-\frac{\gamma}{n}\right|^{2} r^{2}}{n\left(1-\left|1-\frac{\gamma}{n}\right|^{2} r^{2}\right)},
$$

which are valid for each $n \in \mathbf{N}$. Repeated application of (16) and (17) for $n=$ $k, k+1, \ldots, K$ results in the inequality

$$
\begin{aligned}
\frac{1}{k\left(1-\left|1-\frac{\gamma}{k}\right|^{2} r^{2}\right)} & >\sum_{n=k}^{K} \frac{1}{n} \prod_{m=k}^{n-1}\left(\left|1-\frac{\gamma}{m}\right|^{2} r^{2}\right)+\frac{\prod_{m=k}^{K}\left(\left|1-\frac{\gamma}{m}\right|^{2} r^{2}\right)}{K\left(1-\left|1-\frac{\gamma}{K}\right|^{2} r^{2}\right)} \\
& =: s_{k, K}+R_{k, K} .
\end{aligned}
$$

Since $0<R_{k, K}<1 /\left(K\left(1-r^{2}\right)\right)$ and $\lim _{K \rightarrow \infty} s_{k, K}=S_{k-1}$, these inequalities for $k \leq K$ imply the inequality (15). The proof of the theorem is complete. 


\section{References}

[1] Anderson, G. D., M. K. Vamanamurthy, and M. Vuorinen: Conformal invariants, inequalities, and quasiconformal maps. - John Wiley \& Sons, 1997.

[2] Clunie, J. G.: On meromorphic schlicht functions. - J. London Math. Soc. 34, 1959, 215-216.

[3] Clunie, J. G., and F. R. Keogh: On starlike and convex schlicht functions. - J. London Math. Soc. 35, 1960, 229-233.

[4] Duren, P. L.: Univalent functions. - Grundlehren Math. Wiss. 259, Springer-Verlag, New York, Berlin, Heidelberg, Tokyo, 1983.

[5] Gromova, L., and A. VAsiL'Ev: On integral means of star-like functions. - Proc. Indian Acad. Sci. Math. Sci. 112:4, 2002, 563-570.

[6] Jensen, G., and Ch. Pommerenke: On a function-theoretic ruin problem. - Ann. Acad. Sci. Fenn. Math. 32:2, 2007, 523-543.

[7] Libera, R. J.: Univalent $\alpha$-spiral functions. - Canad. J. Math. 19, 1967, 449-456.

[8] Mejia, D., and Ch. Pommerenke: The analytic fixed point function in the disk. - Comput. Methods Funct. Theory 5:2, 2005, 275-299.

[9] Mejia, D., and Ch. Pommerenke: The analytic fixed point function II. - Rev. Colombiana Mat. 40, 2006, 39-52.

[10] Miller, S. S., and P. T. Mocanu: Differential subordinations: Theory and applications. Marcel Dekker, New York, Basel, 2000.

[11] Obradović, M., S. Ponnusamy, and K.-J. Wirths: A proof of Yamashita's conjecture on area integral. - Comput. Methods Funct. Theory 13, 2013, 479-492.

[12] Robertson, M. S.: Quasi-subordination and coefficient conjectures. - Bull. Amer. Math. Soc. $76,1970,1-9$.

[13] Rogosinski, W.: On the coefficients of subordinate functions. - Proc. London Math. Soc. $48: 2,1943,48-82$.

[14] Ruscheweyh, St.: A subordination theorem for $\Phi$-like functions. - J. London Math. Soc. 13, $1976,275-280$.

[15] Solynin, A.: The analytic fixed-point function and its properties. - Zap. Nauchn. Sem. S.Peterburg. Otdel. Mat. Inst. Steklov. (POMI) 337, 2006 (in Russian); Anal. Teor. Chisel. i Teor. Funkts. 21, 238-252, 292; translation in J. Math. Sci. (N. Y.) 143:3, 2007, 3153-3160.

[16] Solynin, A.: Hyperbolic convexity and the analytic fixed point function. - Proc. Amer. Math. Soc. 135:4, 2007, 1181-1186.

[17] ŠPAČEK, L.: Contribution à la théorie des fonctions univalentes. - Časop Pěst. Mat.-Fys. 62, 1933, 12-19 (in Czech).

[18] Temme, N. M.: Special functions. An introduction to the classical functions of mathematical physics. - Wiley, New York, 1996.

[19] Vasil'ev, A.: Univalent functions in two-dimensional free boundary problems, - Acta Appl. Math. 79:3, 2003, 249-280.

[20] Vasil'ev, A., and I. Markina: On the geometry of Hele-Shaw flows with small surface tension. - Interfaces Free Bound. 5, 2003, 183-192.

[21] Yamashita, S.: Area and length maxima for univalent functions. - Bull. Austral. Math. Soc. 41, 1990, 435-439.

Received 1 October 2013• Accepted 25 October 2013 